\title{
Performance Evaluation of the Preanalytic Module of the ACL TOP 750 Hemostasis Lab System
}

Woo-Jae Kwoun, M.D., Jeong-Yeal Ahn 타, M.D., Pil-Whan Park, M.D., Yiel-Hea Seo, M.D., Kyung-Hee Kim, M.D., Ja Young Seo, M.D., Ji-Hun Jeong, M.D., and Hwan Tae Lee, M.D.

Department of Laboratory Medicine, Gachon University Gil Medical Center, Incheon, Korea

\section{Dear Editor,}

Hemolysis, icterus, and lipemia are important preanalytic sources of errors in coagulation testing of blood samples, besides sample-to-anticoagulant ratio, transport stability, and storage temperature. Lipemia (13\%), hyperbilirubinemia (11\%), and hemolysis (4\%) in whole blood cause errors in coagulation testing [1, 2]. Hemoglobin, bilirubin, and triglyceride are major interfering substances that affect test results by spectral overlap [3, 4]. Hemolytic, icteric, and lipemic (HIL) sample check functions have been implemented in the preanalytic module of the ACL TOP family 50 series spectrophotometric hemostasis equipment (Instrumentation Laboratory Company, Bedford, MA, USA) to support preanalytic sample evaluation. We evaluated the performance of the ACL TOP 750 preanalytic module, using patient samples.

In total, 353 blood samples assigned HIL flags by the ACL TOP 750 preanalytic module were collected from samples subjected to the routine hemostasis test. HIL sample flags were given based on absorbance at a specific wavelength. Samples were collected between June and September 2016 and in August 2017 at Gachon University Gil Medical Center, Korea. There were 80 samples with hemolysis flag, 185 samples with icteric flag, 33 samples with lipemic flag, and 55 samples from patients with triglyceride levels over $5,000 \mathrm{mg} / \mathrm{L}$, including samples with- out lipemic flag. Preanalytic values measured by the ACL TOP 750 preanalytic module were compared with reference values obtained from ADVIA chemistry XPT (Siemens Healthineers, Erlangen, Germany) using an enzymatic method and from ADVIA 2120i (Siemens Healthineers, Erlangen, Germany) using a spectrophotometric method. Medians of preanalytic readings were used for analysis. Passing-Bablok regression and Bland-Altman tests were conducted for hemolytic and icteric sample evaluation. Linear regression analysis was conducted for lipemic samples. In each HIL sample group, subgroups of samples with HIL flag and with a value interval around the modal value of the reference method, which included $68 \%$ of all samples with $95 \%$ confidence level, were defined as low-level HIL samples (Table 1).

Passing-Bablok regression for hemolytic samples revealed constant, non-proportional differences between reference and preanalytic values (Fig. 1A). In a Bland-Altman plot, 77/80 (96.3\%) hemolytic and 55/58 (94.8\%) low-level hemolytic samples were within the limit of agreement (Fig. 1B, 1C). For icteric samples, Passing-Bablok regression indicated constant, proportional differences between reference and preanalytic values (Fig. 1D). In a Bland-Altman plot, 174/185 (94.1\%) icteric and 127/137 (92.7\%) low-level icteric samples were within the limit of agreement (Fig. 1E, 1F). The CUSUM test for linearity, indi-
Received: June 25, 2017

Revision received: January 20, 2018

Accepted: April 30, 2018

\section{Corresponding author: Jeong-Yeal Ahn}

(D) https://orcid.org/0000-0001-9842-0748

Department of Laboratory Medicine, Gachon University Gil Medical Center 21 Namdong-daero 774beon-gil, Namdong-gu, Incheon 21565, Korea

Tel: +82-32-460-3831, Fax: +82-32-460-3415

E-mail: jyahn@gilhospital.com

\section{(c) Korean Society for Laboratory Medicine}

This is an Open Access article distributed under the terms of the Creative Commons Attribution Non-Commercial License (http://creativecommons.org/licenses/by-nc/4.0) which permits unrestricted non-commercial use, distribution, and reproduction in any medium, provided the original work is properly cited. 
Table 1. Ranges of preanalytic values and reference values of HIL samples

\begin{tabular}{|c|c|c|c|c|c|c|}
\hline & \multicolumn{2}{|c|}{$\begin{array}{l}\text { Hemolytic sample total/low level } \\
\text { (80/58 samples) }\end{array}$} & \multicolumn{2}{|c|}{$\begin{array}{l}\text { Icteric sample total/low level } \\
(185 / 137 \text { samples })\end{array}$} & \multicolumn{2}{|c|}{$\begin{array}{l}\text { Lipemic sample total/low level } \\
\text { (88/68 samples) }\end{array}$} \\
\hline & $\begin{array}{l}\text { ACL TOP } 750 \\
(\mathrm{~g} / \mathrm{L})\end{array}$ & $\begin{array}{l}\text { ADVIA2120i } \\
(\mathrm{g} / \mathrm{L})\end{array}$ & $\begin{array}{l}\text { ACL TOP } 750 \\
(\mathrm{mg} / \mathrm{L})\end{array}$ & $\begin{array}{l}\text { ADVIA Chemistry XPT } \\
(\mathrm{mg} / \mathrm{L})\end{array}$ & $\begin{array}{c}\text { ACL TOP } 750 \\
\text { (mili absorbance) }\end{array}$ & $\begin{array}{l}\text { ADVIA Chemistry XPT } \\
(\mathrm{mg} / \mathrm{L})\end{array}$ \\
\hline Average & $6.4 / 6.4$ & $5.5 / 4$ & $261 / 259$ & $236.6 / 236.7$ & $1,707.7 / 1,308.7$ & $8.26 / 6.88$ \\
\hline Minimum & $4.3 / 4.4$ & $1 / 3$ & $76 / 210$ & $61 / 186$ & $60.55 / 60.55$ & $1.4 / 3.37$ \\
\hline Maximum & $13.3 / 12.7$ & $28 / 6$ & $428 / 427.5$ & $346 / 296$ & $9,136.8 / 9,168.8$ & $52.26 / 11.87$ \\
\hline
\end{tabular}

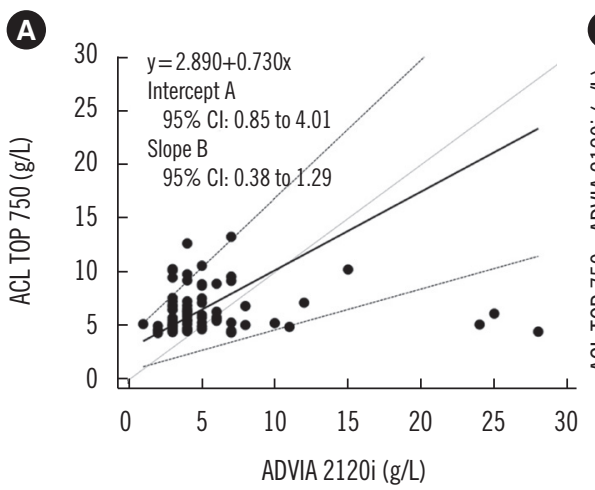

(D)

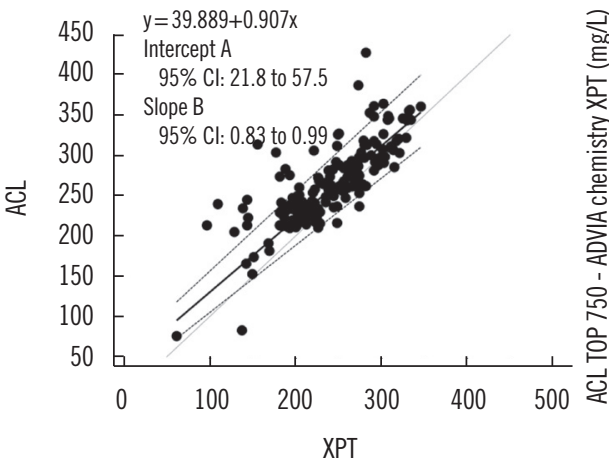

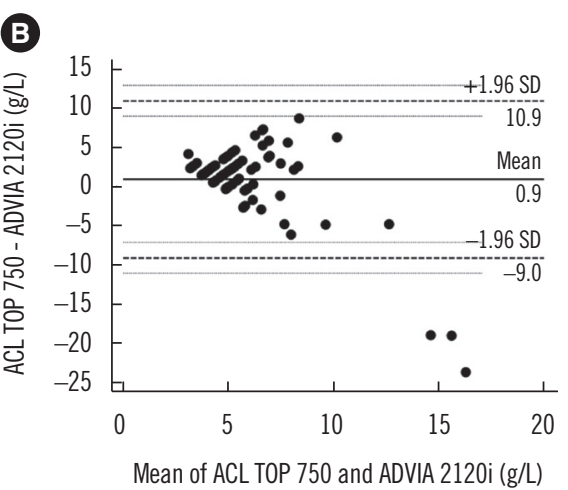

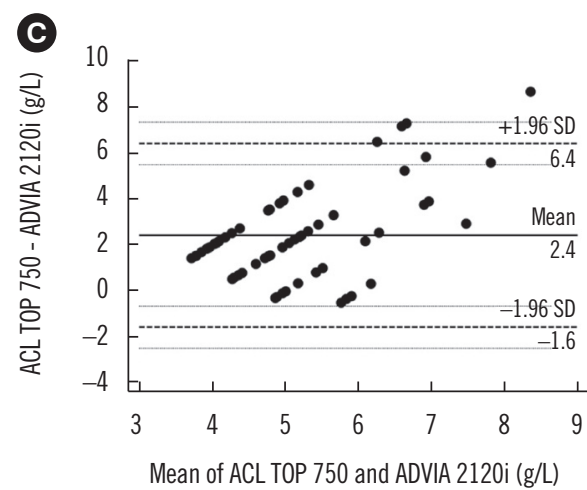

\section{$\boldsymbol{\theta}$}

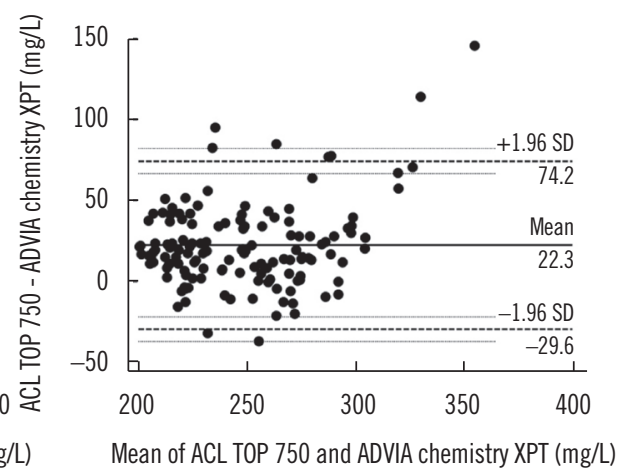

G

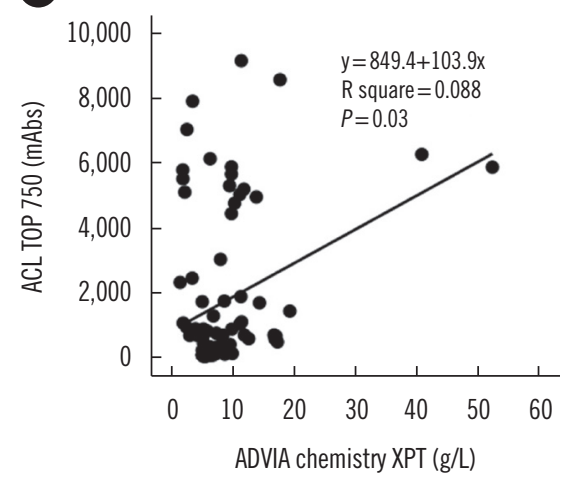

$\boldsymbol{\oplus}$

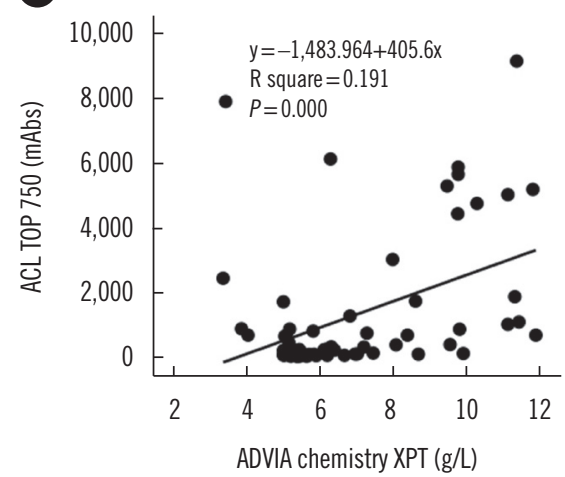

Fig. 1. Statistical analysis result between preanalytic and reference values including $95 \%$ confidence intervals $(\mathrm{Cl})$. (A) Passing-Bablok regression for hemoglobin level in hemolytic sample; the regression equation is $\mathrm{ACL}$ TOP $750=[2.89+0.73 \times \mathrm{ADVIA} 2120 \mathrm{i}]$. (B, C) Bland-Altman plot analysis of total hemolytic sample and low level hemolytic sample. (D) Passing-Bablok regression for bilirubin level of icteric sample; the regression equation is ACL TOP $750=[39.89+0.91 \times$ ADVIA chemistry XPT]. (E, F) Bland-Altman plot analysis of total icteric sample and low level icteric sample. (G, H) Linear regression between absorbance from preanalytic module and concentration of triglycerides from the reference. 
cated a linear relationship between the measurements in both hemolytic ( $P=0.73)$ and icteric samples $(P=0.31)$. Of the $88 \mathrm{li}$ pemic samples, linear regression between preanalytic and reference values indicated no significant association between methods in both total and low-level lipemic samples (Fig. 1G, 1H).

HIL interference was assessed by absorbance measurement of plasma at three wavelengths (405, 535, and $670 \mathrm{~nm}$ ). Interference readings are provided as value ranges on the basis of calculated results, using proprietary algorithms. Manufacturerprovided cut-off values for HIL levels were $5 \mathrm{~g} / \mathrm{L}, 300 \mathrm{mg} / \mathrm{L}$, and 4,885 mili absorbance (mAbs) in the prothrombin time test, and $5 \mathrm{~g} / \mathrm{L}, 280 \mathrm{mg} / \mathrm{L}$, and 4,885 mAbs in the partial thromboplastin time test, respectively.

For hemolytic samples, preanalytic module hemoglobin values tended to be higher than the reference values, and the difference increased with an increase in hemoglobin level. However, only three samples were outside the 95\% limit of agreement (mean $\pm 1.96 \mathrm{SD}$ of the difference), and they showed a high reference hemoglobin level (>20 g/L). For icteric samples, preanalytic values were less influenced by bilirubin concentration and tended to be higher than reference values. However, most of the samples were within the limit of agreement. Therefore, the difference between the measurements of bilirubin is considered acceptable. In lipemic samples, the inter-assay difference in triglycerides could be caused by the different analysis methods employed: preanalytic module with spectrophotometric analysis and reference values obtained by enzymatic analysis. The influence of lipoprotein particles may also vary. The degree of influence is not strongly associated with triglyceride concentration because lipoprotein particle composition and number vary among individuals [5, 6]. Twomey et al [7] noted a weak association between triglyceride and sample turbidity when using intravenous lipid emulsion and a combination of three wavelengths to assess lipemia-induced turbidity.

In conclusion, the ACL TOP preanalytic module can efficiently identify hemolytic and icteric samples, which aids in monitoring sample quality to ensure accurate test results from HIL sample interference and preventing laboratory error in the preanalytic phase. This application allows laboratory technicians to skip inspection of samples, reducing turnaround time, and aids clinicians in accurate sample data interpretation. However, the degree of turbidity of lipemic samples is not strongly associated with triglyceride concentration. Therefore, the term "turbid" instead of "lipemic" would be a more precise term for describing samples analyzed on the basis of absorbance.

\section{Authors' Disclosures of Potential Conflicts of Interest}

No potential conflicts of interest relevant to this article were reported.

\section{REFERENCES}

1. Salvagno GL, Lippi G, Bassi A, Poli G, Guidi GC. Prevalence and type of pre-analytical problems for inpatients samples in coagulation laboratory. J Eval Clin Pract 2008;14:351-3.

2. Salvagno GL, Lippi G, Gelati M, Guidi GC. Hemolysis, lipaemia and icterus in specimens for arterial blood gas analysis. Clin Biochem 2012; 45:372-3.

3. Lippi G, Plebani M, Favaloro EJ. Interference in coagulation testing: focus on spurious hemolysis, icterus, and lipemia. Semin Thromb Hemost 2013;39:258-66

4. Debra AH and JoAnn Molnar. Hemostasis and coagulation instrumentation. In: Rodak BF, Fritsma GA, Keohane E, eds. Hematology: clinical principles and applications. 5th ed. Elsevier Health Sciences, 2013:81028

5. Nikolac N. Lipemia: causes, interference mechanisms, detection and management. Biochem Med (Zagreb) 2014;24:57-67.

6. Kroll MH. Evaluating interference caused by lipemia. Clin Chem 2004; 50:1968-9.

7. Twomey P, Don-Wauchope A, McCullough D. Unreliability of triglyceride measurement to predict turbidity induced interference. J Clin Patho 2003;56:861-2. 